\title{
Revista de la
}

CEPAL

Director

RAUL PREBISCH

Secretario Técnico

ADOLFO GURRIERI

Editor

GREGORIO WEINBERG

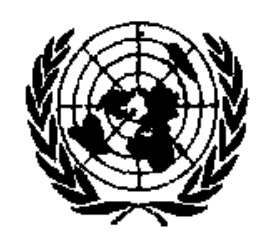

NACIONES UNIDAS

COMISION ECONOMICA PARA AMERICA LATINA

SANTIAGO DE CHILE / ABRIL DE 1980 


\section{S UM A R I O}

El desafio energético

Enrique V. Iglesias

Reunión sobre una nueva América Latina en la cambiante economía mundial

Presentación

Abraham F, Lowenthal y Dovid H. Pollock

Exportación de manufacturas

Pedro I. Mendive

La exportación de productos primarios no combustibles Jere R. Behrman

Una América Latina nueva en el nuevo mercado internacional de capitales Albert Fishlow

América Latina y el sistema monetario internacional observaciones y sugerencias Carlos Massad

Los países latinoamericanos y el Nuevo Orden Económico Internacional Pedro Malán

Desarrollo tecnológico en América Latina y el Caribe Jorge A. Sabato

Las principales cuestiones pendientes en las negociaciones sobre el Código

de Conducta de la UNCTAD para la transferencia de tecnología. Miguel Wionczeck.

La reforma económica internacional y la distribución del ingreso William R. Cline

Resumen interpretativo

Colin I. Bradford, Jr.

Lista de participantes

Repercusiones monetarias y reales de la apertura financiera al exterior.

El caso chileno: 1975-1978

Roberto Zahler

Hacia una teoría de la transformación

Raull Prebisch

Algunas Publicaciones de la CEPAL 


\title{
América Latina y el sistema monetario internacional: Observaciones y sugerencias
}

\author{
Carlos Massad*
}

\section{I \\ El funcionamiento del sistema}

En este trabajo nos proponemos poner de relieve algunos aspectos monetarios y financieros del actual sisstema de relaciones económicas internacionales que dificultan la adecuada inserción de América Latina en la economía mundial. En modo alguno pretendemos realizar un estudio completo de todas las transferencias de recursos entre los paises desarrollados y los paises en desarrollo.

Nuestras observaciones se dividirán en tres secciones principales: el funcionamiento del actual sistema monetario internacional; los efectos del sistema actual en los países latinoamericanos $y$ los paises menos desarrollados en general, y las modificaciones del sistema que podrian contribuir a reducir a un mínimo las actuales dificultades.

A medida que quedó demostrado que el sistema de Bretton Woods no podía ofrecer suficientes incentivos de ajuste a los países de monedas de reserva, en especial a los Estados Unidos y a los países con superávit, la flotación generalizada de las monedas se hizo inevitable. En realidad, la 'disciplina' del FMI sólo podía aplicarse a los países deficitarios que carecian de monedas de reserva. Estos últimos podían financíar sus déficit con su propia moneda, mientras que los países con superávit no necesitaban pedir ayuda al FMI.

La falta de incentivos internacionales para realizar ajustes creó una situación en que no se

*Coordinador del Proyecto Conjunto PNUD-CEPAL, "Implicaciones para América Latina de la situación del sistema monetario y financiero internacional" (RLA) $77 / 021)$

Este documento es una versión revisada de la exposicion realizada por el autor ante la Comisión independiente sobre problemas del desarrollo internacional, el 5 de mayo de 1979 en Annecy, Francia. podia mantener la convertibilidad del oro, y se derrumbó el sistema de Bretton Woods. De hecho, dicha falta de incentivos, unida a ciertas disposiciones bancarias internas, provocó un marcado crecimiento de los mercados financieros privados a medida que aumentó la demanda de intermediación financiera entre los países con superávit y los países deficitarios. Entre 1973 y 1978 el tamaño neto del mercado de euromonedas creció aproximadamente a razón de $19 \%$ al año. De todos modos, estos cambios no sólo son importantes desde el punto de vista cuantitativo sino que expresan, además, una total transformación cualitativa del sistema.

\section{La función que desempeñan las autoridades monetarias y el sector privado}

El primer cambio cualitativo de importancia es el producido en las funciones de intervención en el mercado y de tenencia de reservas de las autoridades monetarias con relación a las desempeñadas por el sector privado. En un sistema de tipo de cambio fijo, las autoridades deben mantener los tipos de cambio a través de la intervención en el mercado, para lo cual deben conservar reservas de divisas, oro y DEG. En un sistema de flotación, dicho papel se traslada total o parcialmente, al sector privado y este último es el que tiene incentivo para acumular 'reservas' para 'intervenir' en los mercados de divisas.

Lo anterior entraña una mayor sensibilidad o elasticidad de la composición de las reservas a los incentivos económicos. Por lo general, los bancos centrales son menos sensibles que los tenedores privados de divisas a los cambios de las expectativas. Cuando se prevé una variación de las tasas de interés o de los tipos de cambio, los últimos tíenden a adaptar rápidamente la composición de sus tenencias. Por lo tanto, mientras más importante sea la función de los tenedores privados en los mercados de divisas, más rápida será la reacción a 
los cambios de las expectativas. Cuando se espera una devaluación de una moneda de reserva $\longrightarrow$ una moneda importante para el comercio internacional-la composición de los activos y pasivos del sector privado cambia inmediatamente, y esto contribuye a provocar la devaluación aguardada. En cierto sentido podría decirse que, de acuerdo con el sistema actual, como los bancos centrales no pueden resistir a las presiones de los especuladores privados, las expectativas generalizadas nunca serán erradas.

Otra consecuencia de la creciente importancia del sector privado en la intervención es la disminución relativa de la demanda de DEG. De hecho, el sector privado no puede mantener DEG por lo que, en la medida en que aumente la importancia relativa de la tenencia privada de monedas de 'reserva', disminuirá la demanda de DEG en relación con la de moneda. Todo esto tiene importantes repercusiones para el sistema en su conjunto (más adelante se profundizarán sus consecuencias para los países menos desarrollados).

a) Las modificaciones de los tipos de cambio tienden a sobrepasar su nivel de equilibrio, de tal modo que los cambios suelen ser de bastante magnitud. Se ha observado que en los países industrializados los precios reaccionan más rápidamente que las cantidades importadas y exportadas a las fluctuaciones del tipo de cambio. Por lo tanto, cuando se devalúa una moneda determinada, poco después tiendem a bajar los precios de las exportaciones y a subir los de las importaciones del país que realiza la devaluación, pero el volumen de las exportaciones y de las importaciones reacciona en forma más lenta, de tal modo que durante un tiempo, la devaluación aumenta el desequilibrio que supuestamente debia corregir. Los tenedores particulares de la moneda verán fortalecidas sus expectativas de devaluación y el tipo de cambio reflejará este hecho con nuevas devaluaciones. Luego, al cabo de un tiempo, el efecto de la devaluación se pondrá de manifiesto en el balance de pagos como una disminución relativa de las importaciones en relación con las exportaciones y se generará el proceso opuesto. Es posible que a medida que se comprenda mejor este proceso y se repita la secuencia de acontecimientos, el sector priva- do 'aprenda' a especular mejor con lo que tendería a desaparecer el efecto desestabilizador de las expectativas formadas en la forma antes señalada. No obstante, se ignora la duración del período de aprendizaje.

b) Se hace más difícil evaluar la rigurosidad de la política monetaria. Por ejemplo, considérese el caso de una empresa con asiento en Alemania, cuyo activo incluya dólares estadounidenses. Si la empresa prevé una baja del valor del dólar en relación con el marco alemán, procurará vender sus dólares por marcos, mientras que si el Bundesbank desea respaldar a lo menos parcialmente el dolar para evitar un fortalecimiento excesivo del marco, comprará los dólares a cambio de marcos. En Alemania, las cifras acusarán una incremento de la oferta monetaria, cuando lo único que ha sucedido es que ha variado la composición de los activos de la empresa domiciliada en Alemania. No hay razón para que esta acción modifique la modalidad de gasto deseada de la empresa, de modo tal que el aumento de la oferta monetaria en Alemania no tendrá efectos inflacionarios directos. Como es natural, el mundo real no es tan sencillo, pero estimamos que el ejemplo sirve para ilustrar la base de nuestro argumento.

c) Los tipos de cambio se hacen más volátiles a través de los cambios que experimentan las transacciones de la cuenita de capital (corrientes de capital). Si los mercados privados de capital y de dinero están bien desarrollados y cuentan con reservas en diversas monedas, las variaciones de las expectativas influirán rápida $y$ plenamente en el mercado: los cambios deseados en la composición de los activos y pasivos de los agentes económicos se reflejarán en acciones en el mercado que, a su vez, se traducirán en modificaciones del tipo de cambio. Los mercados monetarios de las monedas de los países desarrollados son lo suficientemente fuertes como para permitir que funcione un mercado 'a futuro' donde los interesados pueden adquirir 'cobertura' contra los riesgos del tipo de cambio. No obstante, con cobertura o sin ella, a menos que la política en materia de tipos de interés esté estrechamente vinculada con los tipos de cambio esperados, las fluctuaciones de los tipos de interés internos $u$ otros factores que influyen en la composición deseada de los activos y pasivos tenderán a producir 
apreciables movimientos de capital. En la práctica, esta relación es muy dificil de lograr por lo que lo más probable es que los tipos de cambio varíen sensiblemente al cabo de un corto tiempo.

Por otra parte, es posible que los cambios producidos a través de la cuenta de capital tiendan a fortalecer los de la cuenta corriente. Una cuenta corriente desfavorable producirá expectativas de devaluación. Al mismo tiempo, a raíz de la devaluación esperada aumentarán el costo esperado del endeudamiento externo o el beneficio que se espera obtener de las inversiones en el exterior, como consecuencia de lo cual se producirá un desequílibrio de la cuenta de capital de signo análogo al de la cuenta corriente. Como, según se señalo, los ajustes de la cuenta corriente se producen en forma lenta, lo más probable es que, debido a ello, el tipo de cambio varíe en forma relativamente marcada o por lo menos se mueva en un sentido determinado por algún tiempo para luego invertirse en un ciclo que tardará dos o tres años en desarrollarse plenamente.

d) Las marcadas fluctuaciones de los tipos de cambio contribuyen a fomentar tendencias proteccionistas en el comercio mundial. A medida que algunas monedas se valorizan, el pais emisor confrontará un desmejoramiento de sus condiciones de competencia, mientras que los países deficitarios, cuyas monedas disminuyen de valor, tienden a ceder más fácilmente ante los partidarios de adoptar políticas proteccionistas, por razones de balance de pagos. De esta manera, se reduce sustancialmente la resistencia global al proteccionismo, y si al mismo tiempo, por ésta y por otras razones, la economía mundial se expande en forma demasiado pausada, todo se presta para que proliferen los controles directos y las restricciones no arancelarias, $y$ para que se incrementen los aranceles que gravan el comercio.

\section{La transmision de las alteraciones económicas en el plano internacional}

Se esperaba que un sistema de tipos de cambio fijos tendería a igualar las tasas mundiales de inflación, puesto que toda discrepancia produciría desequilibrios en los pagos externos que obligarían al ajuste. No obstante, como los tipos de cambio no eran totalmente fijos, se podría esperar un pequeño margen de discrepancia en las tasas de inflación, por encima de la emanada de las distintas tasas de variación de la productividad en los diferentes países. Entre 1960 y 1970 la tasa de inflación promedio de nueve países industrializados ${ }^{\perp}$ fue de $3.5 \%$, con una desviación estándar de 1.5 y un coeficiente de variabilidad de $0.43 \%$. Se sostuvo que la flotación permitiría que la política monetaria interna fuese más flexible, ya que las fluctuaciones del tipo de cambio pondrían a los países a cubierto de los choques externos. Por estas razones, a partir de 1973 las tasas de inflación deberian haber diferido más que antes, y particularmente más que antes de 1970 .

Sin embargo, las pruebas empíricas no confirman esta expectativa. Entre 1974 y 1976 la inflación anual de los mismos nueve países considerados alcanzó un promedio de $11.3 \%$, con una desviación estándar de $4.8 \%$ y un coeficiente de variabilidad de $0.42 \%$ : es decir, este coeficiente fue prácticamente igual a aquel de los años sesenta, mientras que en el período comprendido entre 1970 y 1973 , que fue de transición, la tasa promedio de inflación fue de $6.5 \%$, con una desviación estándar de 1.7 y un coeficjente de variabilidad de 0.26 . Por lo tanto, en apariencia los tipos de cambio flotantes no contribuyeron mucho a defender a los países de las alteraciones externas. Para explicar este resultado pueden plantearse algunas hipótesis que no es del caso analizar en este documento. Por ahora, basta con señalar que la flotación no ha puesto a los países a cubierto de los choques extemos. La flotación no es un sustituto de políticas internas responsables.

\section{Creación de liquidez internacional}

EI sistema actual ha complicado bastante el concepto mismo de liquidez internacional. Cuando eran las autoridades las que acumulaban la mayor parte de las reservas externas, el concepto era claro. Pero apenas el sector privado asumió en forma parcial o total la responsabilidad de intervenir en el mercado, el concepto de liquidez internacional se hizo vago e impreciso. ¿Debería considerar únicamente las

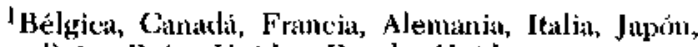
Paises Bajos, Reino Unido y Estados Uuidos.
} 
reservas oficiales? ¿O habria que tomar en cuenta, en alguna forma, las tenencias privadas? Estas preguntas no son tan sólo académicas, puesto que hay una enorme diferencia entre tenencias oficiales y tenencia 'total' de divisas -y oro y DEG- (la segunda duplica por lo menos las primeras).

En un sistema de tipo de cambio flotante, es válido estimar que la tenencia privada de djvisas constituye 'liquidez internacional', puesto que en el sector privado debería existir demanda de dichas tenencias y si ella no es satisfecha, dicho sector buscará formas de hacerlo, aun creando nuevos instrumentos de liquidez. En un mundo donde, por lo general, las operaciones en divisas de los bancos de los paises industrializados y de los centros financieros situados fuera de los países industrializados no están sujetas al mismo tipo de controles que sus operaciones en moneda nacional, la oferta de liquidez internacional llega a determinarse por la demanda. Y en la medida en que las tenencias de divisas del sector privado son un buen sustituto del dinero interno (o nacional), las fluctuaciones de la oferta de las primeras influirán en la demanda del segundo. A través de este proceso los bancos centrales nacionales pierden el control sobre el conjunto de cifras que pueden catalogarse como dinero. Ello no se debe al hecho de que no puedan controlar la oferta de dinero interno a corto plazo, sino a que varía la demanda de dicho dinero, de tal manera que el control o reglamentación no sólo de la tasa de crecimiento de la liquidez internacional, sino también de la liquidez en general, se hace mucho menos efectivo. Como es natural, si la oferta de liquidez en la forma de divisas llega a determinarse fundamentalmente por la demanda, habrá de mermar la importancia relativa de los DEG.

\section{II}

\section{Los efectos en países latinoamericanos y en otros países en desarrollo}

Tal como funciona actualmente el sistema, o la falta del mismo, lo más probable es que los tipos de cambio de las principales monedas tengan fluctuaciones bastante marcadas y reiteradas. La mayoría de los países latinoamericanos, así como otros países menos desarrollados, fijan la paridad de sus monedas con relación a una u otra de las principales monedas o a un conjunto de ellas. Tienen que hacerlo porque la mayoría de ellos carece de mercados financieros o monetarios suficientemente desarrollados como para proceder de otra manera, ya que el Banco.Central es la única entidad capaz de absorber el exceso de oferta o demanda de moneda nacional a corto plazo. Sin embargo, fijar la paridad con relación a otra moneda significa que el valor externo de la moneda nacional sigue el de la moneda con relación a la cual se fija su paridad, y las tuctuaciones de esta moneda se ajustan a las necesidades del país que la emite y no a las del país que la sigue.
Por lo tanto, la flotación entrafia un costo para los países menos desarrollados que se expresa en influencias desestabilizadoras de sus economías. Además, la flotación tiende a desalentar la asignación de recursos adicionales a la producción de bienes comerciables, ya que se introduce un elemento de incertidumbre en todos los cálculos relativos a las actividades asociadas al comercio exterior. En la mayoría de los casos, los exportadores de los países menos desarrollados ni siquiera pueden obtener cobertura porque no existe mercado a futuro para sus propias monedas.

No obstante, hay problemas más serios que éste. Pues, así como la flotación no impide que los paises se vean sacudidos por golpes externos, tampoco resuelve el problema del ajuste a corto o a mediano plazos e incluso puede agravarlo. Es un hecho conocido que si existe un grupo de países que registra un prolongado excedente en cuenta corriente, debe existir otro 
que acuse déficit, y la flotación no corregirá esta situación. A la larga, puede ocurrir que la flotación equilibre el balance de pagos en su conjunto, pero tal vez nunca produzca equilibrio en la cuenta corriente del balance de pagos. Como se vio, de hecho es posible que a corto plazo la flotación de la cuenta corriente del balance de pagos y del balance de pagos en su conjunto, tenga un efecto desestabilizador.

En realidad, el equilibrio de la cuenta corriente no es la situación ideal. Los países en desarrollo no exportadores de petróleo son importadores netos de capital, de manera que sí les conviene que la cuenta corriente acuse déficit, con el que se financiará el superávit de la cuenta de capital. En el caso de América Latina, el déficit promedio en cuenta corriente registrado por los países no exportadores de petróleo entre 1974 y 1978 fue cinco veces superior al del período de 1966 a 1970 . El excedente de capital debería ser lo bastante grande como para cubrir el déficit de la cuenta corriente y el aumento necesario de las reservas. Sin embargo, el superávit de la cuenta de capital es tan sólo una forma más honrosa de referirse a un incremento neto de la deuda externa, ya que las transferencias sin contrapartida son insignificantes y la inversión extranjera directa no aumenta y se concentra en unos pocos paises. En todo caso, el endeudamiento neto reciente representa por lo menos $80 \%$ del superávit de la cuenta de capital de los paises latinoamericanos no exportadores de petróleo.

Pese a los niveles ya alcanzados por dicho endeudamiento (más de 100 mil millones de dólares para los paises latinoamerìcanos no exportadores de petróleo a fines de 1978) para que continúe el proceso de desarrollo y para que los recursos del mundo sean distribuidos de manera más eficiente, dichos niveles deberán seguir elevándose durante muchos años. (Los cuadros 1 y 2 se refieren al financiamiento

Cuadro 1

FINANCIAMIENTO EXTERNO DE LOS PAISES LATINOAMERICANOS NO EXPORTADORES DE PETROLEO

(Miles de millones de dolares)

\begin{tabular}{|c|c|c|c|c|c|c|}
\hline & $\begin{array}{l}1966- \\
1970\end{array}$ & 1974 & 1975 & 1976 & 1977 & $1978+1$ \\
\hline Déficit en cuenta corriente ${ }^{a}$ & -2.0 & -13.1 & -16.1 & -11.5 & -8.0 & -9.5 \\
\hline Aumento de las reservas ${ }^{b}$ & 0.4 & -0.7 & -2.2 & 4.9 & 3.9 & 8.5 \\
\hline Uso de financiamiento externo (usos) & 2.4 & 12.4 & 13.9 & 16.4 & 11.9 & 18.0 \\
\hline Financhamiento extemo neto (fuentes) & 2.5 & 12.6 & 14.4 & 16.2 & 10.5 & 16.0 \\
\hline Inversión directa & 0.7 & 1.6 & 2.3 & 2.2 & (2.3) & \\
\hline Donaciones & 0.1 & 0.1 & 0.1 & 0.2 & 0.2 & 3.0 \\
\hline Préstamos netos & 1.7 & 10.9 & 12.0 & 13.8 & $(8.0)$ & 13.0 \\
\hline Préstamos de fuentes oficiales & 0.9 & 1.9 & 1.9 & $(2.0)$ & $(2.2)$ & 3.0 \\
\hline Multilaterales & 0.4 & 0.9 & 0.8 & $(0.9)$ & (1.0) & \\
\hline Bilaterales & 0.5 & 1.1 & 1.0 & (1.1) & (1.2) & \\
\hline Créditos de tuentes privadas & 0.8 & 9.0 & 10.1 & 11.8 & (5.8) & 10.0 \\
\hline Créditos de los abastecedores & 0.4 & 0.2 & 0.1 & 0.6 & $(0.6)$ & 1.5 \\
\hline Bancos comerciales & 0.3 & 8.2 & 8.2 & 7.5 & 4.7 & 5.5 \\
\hline Bonos & - & 0.1 & 0.2 & 0.5 & $(1.0)$ & 2.0 \\
\hline Otros y sin asignar & 0.1 & 0.6 & 0.6 & 2.2 & -0.5 & 1.0 \\
\hline
\end{tabular}

Fuentes: Fondo Monetario lnternacionul, Balance of Payments Yearbook; Bank for International Settlements: Suplementos de julio y diciembre de 1978 y Yearbook; estinaciones de la CEPAL.

- Excluye las donaciones oficiales.

D El signo positivo indica un incremento de las reservas.

- Incluye préstamos no compensatorios y compensatorios a largo, a mediano y a corto plazo.

d Todas las cifras correspondientes a 1978 son provisionales. 
Cuadro 2

ESTIMACION DEL ENDEUDAMIENTO GLOBAL DE LOS PAISES

LATINOAMERICANOS NO EXPORTADORES DE PETROLEO

(Miles de millones de dólares)

\begin{tabular}{lccc}
\hline Años & $\begin{array}{c}\text { Deuda garantizadia } \\
\text { por el Estaclo }\end{array}$ & $\begin{array}{c}\text { Deuda a los bancos } \\
\text { no garantizada }\end{array}$ & Endeudamiento globali" \\
\hline 1974 & 31.51 & 19.76 & 51.72 \\
1975 & 38.05 & 24.50 & 63.48 \\
1976 & 48.74 & 30.65 & 81.28 \\
1977 & 59.00 & 32.00 & 91.00 \\
1978 & 68.00 & 37.00 & 106.00 \\
\hline
\end{tabular}

- Incluye la deuda con el FMI.

b Lats cifras correspondientes a 1978 son provisionales.

y al endeudamiento globales de los países latinoamericanos no exportadores de petróleo.) Sin embargo, la inestabilidad adicional a corto plazo del balance de pagos, que es un subproducto de la flotación, no facilita a los paises industrializados la adopción de decisiones oficiales sobre el financiamiento a largo plazo de desarrollo, mientras que los mercados financieros privados se expanden rápidamente. Por lo tanto, las condiciones de la nueva forma de financiamiento son bastante peores que las del pasado, tanto en lo que respecta a los tipos de interés como a los plazos de amortización. La 'carga de la deuda' se convierte en un problema para obtener nuevos préstamos, problema que es más bien consecuencia del sistema actual y no del 'mal comportamiento', aunque también suela darse éste. Por otra parte, a medida que se deterioran las condiciones de la deuda, los países prestatarios deben aumentar sus reservas tanto para presentar una mejor 'imagen' como para estar en condiciones de neutralizar las posibles salidas de capital. Así, pues, a medida que empeoran las condiciones, tiende a crecer la tasa necesaria de acumulación de reservas, y lo mismo sucede con el endeudamiento anual neto requerido.

Una de las maneras obvias en que los países menos desarrollados podrían resolver este problema sería aumentando las exportaciones. Si éstas crecieran a un ritmo acelerado, podrían reducirse tanto la 'carga de la deuda' como el déficit de la cuenta corriente. No obstante, como ya se señaló, en las actuales circunstancias los países desarrollados tienden a ceder más fácilmente a las presiones proteccionistas, de tal manera que esta solución no parece factible. De hecho, un artículo publicado recientemente en el Boletin del FMI llega a la conclusión de que el proteccionismo ha aumentado últimamente de manera significativa y que la tendencia no lleva visos de declinar. ${ }^{2}$ Cabe lamentar que esta tendencia haya surgido precisamente ahora que varios países menos desarrollados, a lo menos de América Latina, aplican una estrategia de apertura al exterior.

Para que esta estrategia logre éxito tendría que satisfacer dos requisitos previos: contar con mercados en el extranjero y disponer de financiamiento externo. Los primeros son objeto de creciente protección de la competencia externa. El último puede obtenerse, pero en condiciones que sólo son compatibles con un crecimiento acelerado de las exportaciones de los países menos desarrollados.

2"La liberalización del comercio pierde terreno, pues se intensificun las medidas restrictivas", Boletin del FMI, 16 de abril de 1979 . 


\section{III}

\section{¿Qué puede hacerse para resolver, o por lo menos atenuar las dificultades con que tropieza actualmente el sistema monetario y financiero?}

Al menos algunas de las causas de las dificultades antes señaladas pueden atribuirse a problemas propios del proceso de ajuste y de la creación de liquidez. Si el proceso de ajuste marchara sin tropiezos y la liquidez internacional creciera a una tasa razonablemente estable, desaparecerian las tluctuaciones excesivas del tipo de cambio y no se debilitaría tanto la voluntad para resistir las medidas proteccionistas.

Podría sostenerse que el proceso de ajuste marcha bien cuando optar entre un tipo de cambio fijo o uno flotante llega a carecer de ímportancia. En otros términos, si se coordinaran estrictamente las políticas internas de los principales países industrializados no habría que modificar sus tipos de cambio entre sí a corto plazo y, por lo tanto, la flotación sería innecesaria. Espero que a estas alturas resulte evidente cuán importante es para los países menos desarrollados que los tipos de cambio sean más estables y el proceso de ajuste más parejo.

Pero, naturalmente, la estrecha coordinación de las políticas internas es un ideal muy difícil de alcanzar. Los diversos paises tienen instituciones, grupos de intereses y fuerzas políticas y sociales diferentes. Por ejemplo, algunos paises pueden exportar a sus desempleados $y$, por lo tanto, están en mejor situación que otros de aceptar políticas económicas más restrictivas.

No obstante, por dificil que sea, estimo que habría (jue seguir tratando de asegurar una coordinación más estrecha de las políticas económicas entre los paises industrializados. Como es natural, dicha coordinación debería tener presente las necesidades globales, reconociendo el déficit de cuenta corriente de los países en desarrollo y facilitando su financiamiento. De esta manera, se lograría que el proceso de transferencia de los recursos reales fuese más armónico. Para asegurarse de que se tendrán en cuenta las necesidades globales, los países menos desarrollados deberían participar de alguna manera en las discusiones sobre coordinación de políticas que tienen lugar entre los países industrializados.

En esta materia desempeñan un papel importante los informes que ofrecen una visión global de la economia mundial. Por ejemplo, debería dárseles mayor difusión a los excelentes informes periódicos del FMI sobre las perspectivas económicas mundiales. Tal vez el comité interino de la Junta de Gobernadores del Fondo debería consagrar por lo menos una sesión completa durante el año a evaluar y analizar la situación económica mundial. Sin embargo, en muchos países la coordinación de políticas hiere puntos muy sensibles y por esta razón exige cierto grado de participación formal de los gobiernos en el más alto plano político.

No obstante, para todos los países, tanto en desarrollo como desarrollados, es importante mejorar el proceso de ajuste.

Todos los esfuerzos que se destinen a alcanzar la meta de coordinación de las políticas valen la pena. Sin embargo, no puede esperarse que por sí solos tales esfuerzos sean plenamente efectivos por lo que aun antes de mejorar la coordinación hay que adoptar algunas medidas en campos particularmente difíciles:

a) Debería establecerse el pago de las obligaciones internacionales con activos, a fin de crear un incentivo de ajuste en los países de moneda de reserva. Si lo corriente fuese pagar con activos, los países no podrían cumplir sus compromisos internacionales símplemente aumentando su pasivo en el extranjero. Por lo tanto, si un país de moneda de reserva incurriere en déficit, lo cubriría con activos, como cualquier otro pais. El incentivo en favor del ajuste aparecería a medida que se agotan tales activos.

b) Debería crearse en el FMI una cuenta para sustituir las monedas de reserva por DEG. 
Su función consistiría en reducir al mínimo las presiones de que son objeto los tipos de cambio como consecuencia de las modificaciones que las autoridades monetarias desean introducir en sus carteras de divisas. Los países emisores de la moneda acumulada en la cuenta la recuperarían dentro de un plazo convenido, a cambio de DEG. En realidad, ésta sería una forma en que los países industrializados podrían consolidar su deuda a corto plazo. A medida que estos países recuperen la moneda que tienen en la cuenta, los DEG alli acumulados podrian utilizarse para otorgar préstamos a largo plazo a los países menos desarrollados. Esta operación podría denominarse 'vínculo de sustitución".

c) Los países cuyas reservas netas excediesen, por ejemplo, las importaciones correspondientes a cuatro meses y aumentasen a un ritmo más acelerado que una determinada tasa anual pagarian un impuesto sobre sus reservas. Una de las formas de aplicar este concepto sería, por ejemplo, no asignar DEG a tales países en una futura distribución, destinándose los DEG no asignados a ellos a los países menos desarrollados en proporción a sus cuotas en el FMI. De esta manera, se alentaría a los países con superávit a realizar el ajuste. Esto podría llamarse 'vínculo de ajuste'.

d) Debería establecerse un servicio de refinanciamiento de la deuda, quizá como empresa conjunta del Banco Mundial y del FMI. El servicio funcionaría con arreglo a un sistema similar al del servicio del petroleo del FMI. Los países en desarrollo tendríar acceso a él, a voluntad, sobre la base de un conjunto de indicadores preestablecido, pero el monto y condiciones del refinanciamiento se estudiaria caso por caso. El servicio de refinanciamiento sería una contrapartida natural de los paises menos desarrollados a una cuenta de susti- tución para los países de moneda de reserva.

e) Debería establecerse un foro para analizar periodicamente $y$ en forma conjunta los problemas monetarios del comercio y del desarrollo, que están tan estrechamente vinculados, destacándose las principales tendencias y medidas de política sugeridas. Dicho foro podria evaluar la contribución global al desarrollo de cada país industrializado teniendo presente el aporte realizado a través del comercio y a través de la ayuda y otras corrientes financieras. Lo ideal sería crear algún tipo de tribunal económico internacional que se pronunciara, en especial, sobre las prácticas comerciales restrictivas, y estableciese una indemnización por los daños económicos ocasionados. Los países podrían entonces apreciar si les conviene o no aplicar medidas proteccionistas y pagar indemnización por ellas. Este concepto de 'proteccionismo indemnizado' podría perfeccionarse aún más como una manera de permitir cierta libertad a los países al respecto, pero contemplando pagos compensatorios a los países perjudicados en el proceso. La multipolaridad del mundo actual imparte cierto realismo a esta propuesta.

Como es natural, la mayoría de estas ideas no son ni nuevas ni realistas. Sin embargo, el problema del desarrollo habrá de acompañarnos por un tiempo lo suficientemente prolongado como para permitimos aplicar en este momento algunos criterios poco realistas. A nuestro juicio, a medida que pase el tiempo, se hará cada vez más evidente que el desarrollo no es un problema que atañe exclusivamente a los países en desarrollo, sino al mundo en general. En ello nos basamos para considerar no sólo los problemas de los países latinoamericanos, o de todos los países en desarrollo, sino también los que aquejan a la economía mundial con los cuales estos últimos países se encuentran tan estrechamente vinculados. 